\title{
Voluntary forgetting before and after learning has been accomplished
}

\author{
SAM S. RAKOVER* \\ University of Haifa, Haifa, Israel
}

\begin{abstract}
Two experiments, in which voluntary forgetting was measured, are reported. In both experiments, lists of randomly mixed syllables and numbers were projected on a screen, and Ss were instructed before projection which part of the lists to remember or to forget. In the first experiment, the syllables were cued as items to be forgotten in one group, and to be remembered in the other one. At the end of the projection, Ss were instructed to recall freely only the syllables. For the former group, this was an unexpected test. The same list was projected for different groups: one, three, five, seven, and fourteen times, with new randomly mixed items each time. Syllable recall increased with an increase of times of projection of the list in the second group, but not in the first one. Recall of syllables in the first group was, however, above the chance level. These results were best interpreted by assuming a voluntary forgetting mechanism, which operates after the verbal items leave the short term store. The second experiment, a variation of the first, was designed to find the locus of voluntary forgetting mechanisms. The question is whether Ss can voluntarily forget syllables only before they are learned, or also after learning has already taken place. Voluntary forgetting after learning has been achieved was found to be ineffective. These results tend to support the hypothesis that voluntary forgetting processes do not operate in the long-term store and that Ss can block the transmission of to be forgotten items from the short-term store to the long-term store.
\end{abstract}

Voluntary forgetting has been extensively studied in a short-term memory experimental paradigm, see reviews by Bjork (1972) and by Epstein (1972). This paradigm is operationally defined as the recall of verbal items, which are shown only once, for a short period of 1 to $3 \mathrm{sec}$. In accordance with this method, Ss in the experimental group were instructed that some items were to be forgotten (TBF items) and others were to be remembered (TBR items), while the control group did not receive any voluntary forgetting instructions. Recall of TBR items was better in the experimental group than in the control group. Recall of TBF items in an unexpected test, although above the chance level, was much less accurate than recall of TBR items. These results support the general hypothesis that, in a short-term memory experiment, Ss can voluntarily forget verbal information when they are instructed to do so. The question is whether Ss can voluntarily forget verbal information in a long-term memory experimental paradigm. This paradigm is defined operationally as the recall of verbal information, which is shown several times, for a long period. A negative answer to the above question means that Ss cannot avoid learning verbal information against their will.

\section{EXPERIMENT I}

\section{Method}

Materials and Equipment. Each stimulus consisted of a black-on-white photograph of one typewritten CVC trigram nonsense syllable (sy.s) or one 3-digit number (no.s), projected

\footnotetext{
*Special thanks to H. Kaminer for helping to conduct the experiments and to $B$. Beit-Hallahmi for reading the manuscript and making helpful comments and suggestions. This research was funded through the University of Haifa.
}

from a Kodak carousel slide projector. There were two lists, each composed of sy.s mixed randomly with no.s. The first list, the practice list, included three different sy.s and three different no.s. The second list, the test list, was comprised of nine new sy.s and nine new no.s. Each list began with a blank slide and ended with an asterisk. The sy.s were taken from Underwood and Schulz (1960, Appendix A, the Glaze's sy.s, Column 1, range $80-100$ ). All the sy.s were examined by 40 psychology students to exclude those having sounds similar to words in Hebrew or Arabic. The remaining syllables were given to 36 other psychology students in order to rank each sy.s on a five-point scale of subjective association value. (Instructions were similar to those of Noble, Stockwell, \& Pryer, 1957). From those sy.s ranked with the highest average subjective association value, 12 were randomly chosen for the practice and test lists.

Design. Ten groups were tested according to a 2 by 5 factorial design. The first factor was the instruction to remember or forget the no.s or the sy.s, and the second factor was the number of times the same test list was shown. Sixty-two Ss participated in the remember:no.s - forget:sy.s groups. (R:no.s-F:sy.s). Before projection of the test list, these $S s$ were instructed to remember the no.s and to forget the sy.s. They were also informed that they would be tested only on the no.s. Forty other Ss were instructed to remember the sy.s and to forget the no.s (R:sy.s-F:no.s). They were informed that they would be tested only on the sy.s. After the test list was projected, however, all Ss in all groups were instructed to recall only the sy.s. For the R:no.s-F:sy.s groups this was an unexpected test. The same test list was projected one, three, five, seven, or fourteen times. Each time the same items were mixed randomly in a different order. Random mixing of the list items ensured that Ss would observe every item, whether it was TBF or TBR. These 10 independent groups ( 2 group ty pes by 5 times of list exposure) are designated as follows: R:no.s-F:sy.s(1), $(n=12) \ldots R:$ Ro.s-F:sy.s(14), $(n=14) \quad R: s y . s-F: n o . s(1)$, $(n=8) \ldots R:$ sy.s-F:no.s $(14),(n=8)$.

To these ten groups, the following three new groups were added as controls for the effect of the forget instructions on sy.s recall. These instructions could create several differences in Ss' behavior which could differentially influence recall of the sy.s. Ss might avert their gaze or close their eyes after the perception of the TBF items, and thus observe these items for a shorter time (less than $1 \mathrm{sec}$ ). In order to check this possibility, two groups 
were run as a comparison to the R:no.s-F:sy.s(1) group. Ss in the $R:$ no.s-F:sy.s(1, open) group, $n=12$, were requested to keep looking at the TBF items, in order to improve perception of the TBR items, with which they were randomly mixed. Ss in the $R: n o . s-F$ :sy.s(1, closed) group, $n=12$, were requested to close their eyes after perceiving the TBF items, in order to improve forgetting of these items. They were also instructed to open their eyes on hearing a buzz, which would signal the end of the TBF item exposure in order to perceive the next item (without the signal it was hardly possible to perform this task).

$S s$ in the remember sy.s condition might rehearse these items, while those in the forget sy.s condition might block this behavior. In order to check on this possibility the R:sy.s-F:no.s $(14$, no rehearse) group, $\mathrm{n}=8$, was run as a comparison to the R:sy.s-F:no.s (14) group. In the former, a new group, Ss were instructed not to rehearse the TBR items in the interslide interval and while the TBF items or a blank slide were exposed.

Procedure. Each item on the practice list and test list was projected onto the screen for $1 \mathrm{sec}$. The interslide interval, which was also the intertest lists interval, was $.55 \mathrm{sec}$. The lists were projected by two Kodak carousel projectors programmed successively. Ss were not informed that the first list was a practice list. Prior to projection of the first list, Ss were informed that they would see a list of mixed no.s and sy.s. They were also informed that after the presentation of the list, they would recall items only when instructed to do so by the $E$. This was important for those groups which would have to recall the TBF items unexpectedly, and for those groups who would see the same test list several times. Ss received $1 \mathrm{~min}$ for free recall of the practice list. After this period, the $E$ informed the $S$ s that they were about to see another list of randomly mixed no.s and sy.s (the test list), which would be exposed for several times, each time with different random orders of items. He then instructed the Ss which parts of the list they would memorize or forget, as described in the design. At the end of the test list projections, Ss received $90 \mathrm{sec}$ for free recall. After this, they were asked to complete a questionnaire concerning the amount of attention paid to the TBF items, or rehearsal of the sy.s items. Ss were tested in groups of three or four and were assigned to participate in the experimental situation at the time they volunteered. The order of the projection of the same test lists, with different randomly mixed items was also randomly changed before each new group of Ss.

Subjects. One-hundred and thirty-four undergraduates volunteered to participate in Experiment I. One hundred and two Ss were in the main 10 experimental groups and $32 \mathrm{Ss}$ were in the three control groups. Average age and sex composition were kept constant in these groups.

\section{Results}

Only recall of correct sy.s on the test list are reported. Ss in each of the 10 experimental groups: R:no.s-F:sy.s and in the R:sy.s-F:no.s, were separated into two subgroups on the basis of the postexperimental questionnaire. One subgroup was comprised of those subjects who suspected that they would be requested to recall TBF items; the other was comprised of those Ss who did not suspect. A Newman-Keuls test at $\alpha=.05$ (Winer, 1971), showed that each of the subgroups in the R:sy.s-F:no.s recalled more syllables than the subgroups of the R:no.s-F:sy.s. There were no significant differences, however, between these subgroups in recall of sy.s within the R:sy.s-F:no.s or the R:no.s-F:sy.s groups. The statistical analysis to be reported was therefore made on all Ss.

There were no significant differences in sy.s recall among these groups: R:no.s-F:sy.s(1); R:no.s-F:sy.s(1, open) and R:no.s-F:sy.s(1, closed).
A postexperimental questionnaire revealed that none of the eight Ss in the R:sy.s-F:no.s(14, no rehearse) rehearsed the sy.s, as they were instructed to do so. No significant difference in sy.s recall was found between this group and the R:sy.s-F:no.s(14).

Figure 1 shows the mean of sy.s correctly recalled expressed as a function of the group types and the number of times the test was exposed in Experiments I and Il. A 2 by 5 analysis of variance (Winer, 1971) revealed that the R:sy.s-F:no.s groups recalled more sy.s than the R:no.s-F:sy.s groups, $F(1,92)=259.13$, $p<.01$. Syllables recall increased as the number of test list exposures increased in the R:sy.s-F:no.s groups, but not in the R:no.s-F:sy.s groups, $F(4,92)=6.76, p<.01$. A single analysis of variance made on R:no.s-F:sy.s groups revealed no significant differences among them. Recall of sy.s in the R:no.s-F:sy.s groups was, however, above the chance level, as tested by the Poisson distribution at $\alpha=.01$. A $t$ test, based on the $F$ test, showed that the R:sy.s-F:no.s(1) group recalled significantly more sy.s than the R:no.s-F:sy.s(1) group, $t(92)=6.43, p<.01$. This last result is in accordance with the findings reported in the literature (Bjork, 1972; Epstein, 1972). Ss do voluntarily forget verbal information in a short-term experimental paradigm.

\section{Discussion}

The result that there are no significant differences among the R:no.s-F:sy.s (1, open); R:no.s-F:sy.s (1, closed) and the R:no.s-F:sy.s(1) groups, made it unlikely, that the possibility of averting the gaze or closing the eyes, after perception of the TBF items caused the differences found between the forget and remember sy.s conditions. This conclusion is also supported indirectly by the results that there are no significant differences in sy.s recall between Ss who reported suspicion on being tested on TBF items and those who did not.

The possibility of interpreting the results, by assuming rehearsal of the sy.s in R:sy.s-F:no.s situation and not in the R:no.s-F:sy.s situation is not supported. First, there was no significant difference in sy.s recall between the R:sy.s-F:no.s(14) and the R:sy.s-F:no.s (14, no rehearse) groups. Second, although $\mathrm{Ss}$ in the forget sy.s situation probably did not rehearse the TBF items, nonrehearsing could not explain the flat curve of sy.s recall as a function of number of list projections found in the R:no.s-F:sy.s groups. Rehearsal means that Ss keep introducing the items repeatedly in the short-term store (STS) and by that increase their recall (Bjork, 1972). The same thing, or even better, can be done by repeatedly projecting the same list. Thus, even if rehearsal was blocked, one should predict that the recall in the R:no.s-F:sy.s groups, should increase with the number of test list projections-a prediction that was not sustained. To take an extreme example, it is hard to see how nonrehearsing would explain the insignificant differences in the same list recall, between the $\mathrm{R}$ :no.s-F:sy.s group to whom the list was exposed only 


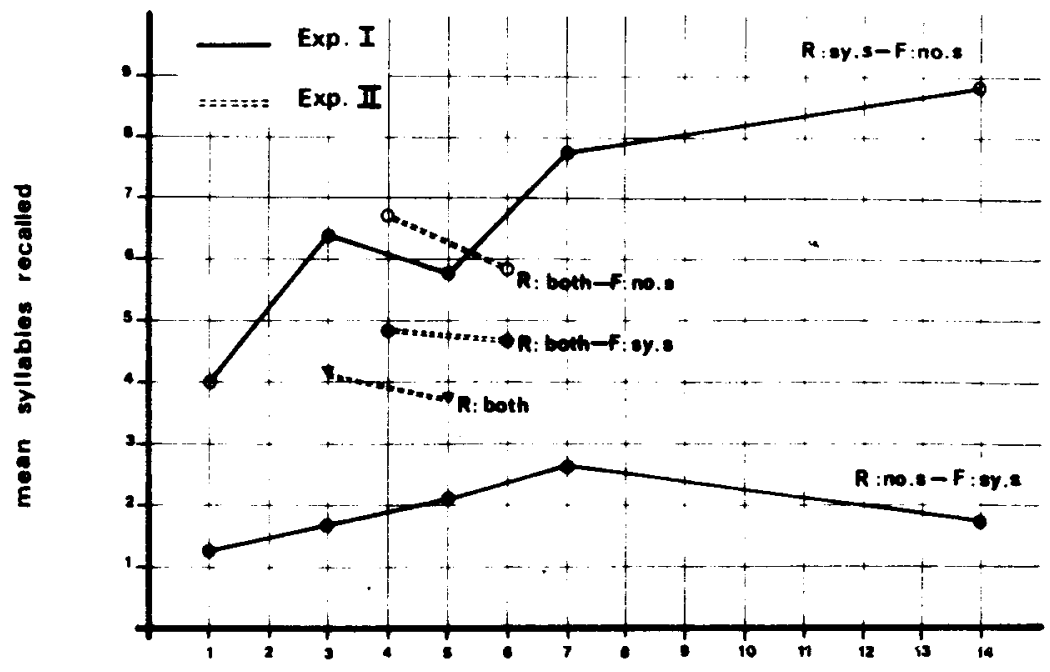

Fig. 1. Mean syllables recalled as a function of number of test list projections. once and the group to whom the list was exposed fourteen times. Third, if rehearsal is responsible for correct sy.s recall, and if this process was blocked in the forget sy.s condition, how did SS recall sy.s above the chance level? Moreover, if rehearsal was not blocked completely, one should predict an increase in sy.s recall in the forget sy.s condition-a prediction that was not sustained.

Several other researchers reached the conclusion that rehearsal has insignificant effects in short term memory experiments of voluntary forgetting (Bjork, LaBerge and Legrand, 1968; Block, 1971; Shebilske, Wilder, \& Epstein, 1971; Epstein, Massaro, \& Wilder, 1972).

It seems that in a long-term memory experiment Ss could not avoid learning verbal information to some degree although they were instructed not to do so. Since there were no significant differences among all the groups in the forget sy.s condition, one might conclude that Ss in the long-term memory groups recalled the same amount recalled in the short term memory group-the F:sy.s-F:no.s(1) group.

The results of this experiment can best be interpreted by assuming a voluntary forgetting mechanism, which is operating on the verbal information after the items leave the STS. This mechanism could be located in the following stages: in the transmission of the information from the STS to the long term (LTS), in the LTS or in the retrieval stage. It cannot be located in the stage before the information reached the STS or in the STS, since Ss recalled one to three correct sy.s in each one of the forget sy.s groups. According to this hypothesis, one should expect that sy.s recall in the forget sy.s groups should be from the STS alone, while in the remember sy.s groups recall would be from both stores. In the former groups this would cause recall of one to three sy.s from a limited capacity information STS, while sy.s recall would increase with the number of the test list projections in the latter groups.
The question which arises is whether Ss can voluntarily forget verbal information when it has already been encoded in the LTS, that is, after learning has already been accomplished, or do Ss have to forget them before the information has entered the LTS?

\section{EXPERIMENT II}

Experiment II designed to answer the questions raised in Experiment I, was based on the following three stages: In the first stage Ss learned the TBF items; in the second stage they were instructed to erase TBF items completely from their memory, that is, to forget TBF items after already having learned them; and in the third stage Ss were requested to recall these items on an unexpected test. The results of this experiment are compared to those of Experiment I where instructions to forget were given before exposure of the verbal information. If recall of TBF items in Experiment II is greater than in Experiment $I$, it can be concluded that voluntary forgetting does not operate in the LTS. Other results of this comparison would support the conclusion that voluntary forgetting might operate in short term as well as in long term memory.

\section{Method}

Design and Procedure. This experiment was identical to Experiment I except for the following: 24 new Ss participated in the remember both no.s and sy.s and afterwards remember no.s and forget sy.s group (R:both-F:sy.s). They were informed that the test list would be projected several times. Before projection of the test list for the last time, they were informed which items (no.s or sy.s) would be TBR and TBF. Up to this point Ss had to memorize both the no.s and sy.s, since they would not know which part of the test list would be TBR or TBF. At this point, however, while viewing the test list for the last time, they would have to memorize the TBR items only and to completely erase TBF items from their memory. For this group, TBR items were the no.s; TBF items were the sy.s. At the conclusion of the last test list projection, Ss were instructed to free recall the sy.s only. 
For the R:both-F;sy.s group this was an unexpected test. Sixteen other students participated in the remember both no.s and sy.s and afterwards remember sy.s and forget no.s group (R:both-F:no.s). This group was identical to the R:both-F:sy.s group, except that before exposure to the test list for the last time, Ss were instructed to remember sy.s and forget no.s. They were also instructed to free recall the sy.s only.

As a control for these two groups, another 16 students participated in the remember both no.s and sy.s group (R:both) Ss were instructed to remember both no.s and sy.s, since they would be informed which part of the list was TBR or TBF only after the projection of the test list for the last time. The test list was shown several tiems, after which the $\mathrm{Ss}$ were requested to free recall the sy.s only.

In the $\mathrm{R}$ :both-F:no.s and $\mathrm{R}$ :both-F:sy.s groups, the test list was projected three or five times, not including the last exposure. In the $\mathrm{R}:$ both group the test list was projected three times or five times only. These six groups ( 3 sets of instruction by 2 number of times of list projections) are designated as R:both (3) ... R:both-F:no.s(6) ... and R:both-F:sy.s(6).

Subjects. Fifty-six undergraduates participated in the second experiment. There were $12 \mathrm{Ss}$ in each of the R:both-F:sy.s groups and $8 \mathrm{Ss}$ in each of the four other groups. Average age and sex composition were kept constant in these groups.

\section{Results}

As in Experiment I, there were no significant differences in sy.s recall between Ss who suspected that they would be requested to recall TBF items and those who did not.

Efficiency of voluntary forgetting is markedly reduced if the instructions to forget are given after learning (see Fig. 1). This conclusion is based on the following results: first, $S$ s in each of the R:both-F:sy.s $(4,6)$ groups recalled more sy.s than subjects in each of the R:no.-F:sy.s $(3,5,7,14)$ groups taken from Experiment $\mathrm{I}(\mathrm{p}<.01$ for each $\mathrm{t}$ test comparision); and second, there were no significant differences between the R:both-F:sy.s $(4,6)$ and the R:both $(3,5)$ control groups.

The last test list exposure improves sy.s recall in the R:both-F:no.s groups, while it has no effect on the $\mathrm{R}$ :both-F:sy.s groups. This conclusion is based on the following results: first, $S$ s in the R:both-F:no.s groups recalled more correct sy.s than $S$ s in the $R$ :both-F:sy.s groups, which recall as the $R$ :both control groups $F(1,36)=11.60, p<.01$; and second, there were no significant differences in sy.s recall among the R:both-F:no.s $(4,6)$ and the R:sy.s-F:no.s $(3,5)$ groups taken from Experiment I.

\section{DISCUSSION}

It could be hypothesized that Ss in Experiment I in the R:no.s-F:sy.s groups recalled fewer sy.s than $S$ s in the R:sy.s-F:no.s groups, because they were complying more with instructions to forget sy.s than with the unexpected requirement to recall them. According to this hypothesis, there should be no significant differences between the R:both-F:sy.s groups and the $R:$ no.s-F:sy.s groups, since $S s$ in both cases should comply more with instructions to forget the sy.s than to recall them unexpectedly. The results do not support this hypothesis.
In view of the findings of these two experiments, where does the voluntary forgetting mechanism operate in the following stages of the verbal information processes? These mechanisms could be located in the transmission of the information from the STS to the LTS, in the LTS, or in the retrieval stage. It is difficult to locate the voluntary forgetting processes in the retrieval stage, where Ss selectively search for the TBF items (Epstein, 1972), since the search set and its composition were kept constant. The number of the TBF items, which was equal to the number of the TBR items, was kept constant throughtout the experiments.

The results also do not support another quality of the selective search hypothesis, namely, the assumption that TBF and TBR items availabilities are equal (Epstein \& Wilder, 1972). There were significant differences in sy.s recall between the remember and forget sy.s groups of Experiment I. Since the following explanations of this finding-closing the eyes, rehearsal, and search set-did not fit the results, the only explanation left was that of encoding the TBR differently from the TBF items, that is, in their differing availability. The differences in sy.s recall found between Experiment I and Experiment II in the forget sy.s groups supports this conclusion.

The locus cannot be in the LTS since Ss could not forget the TBF items after learning had already taken place. Their recall of sy.s was superior to that of Ss who were instructed to forget the TBF items before the list projection, and in addition, there were no significant differences between them and $S$ s in the control group.

In summary, the forgetting processes mechanism does not seem to operate before and in the STS (Experiment I), in the LTS or in the retrieval stage (Experiments I and II). Thus the only stage left for this mechanism to operate, is in the transmission of information from the STS to the LTS. This suggests a new hypothesis, called the "blocking" hypothesis, according to which SS control the transmission of verbal information from the STS to the LTS: they can block one type of information (TBF items) while letting a second type pass (TBR items). From this hypothesis it can be implied that $S s$ in the R:no.s-F:sy.s groups retrieved the TBF items from the STS only. This amount of sy.s recall should be independent of the number of the test list projections.

The "blocking" hypothesis could also offer an explanation to the significant difference in sy.s recall between the R:both-F:no.s and R:both-F:sy.s groups. In the latter group Ss blocked the transmission of verbal information from the STS to the LTS in the last test list exposure, while not so in the former groups, since just before this trial they were informed that the sy.s would be the TBF items.

What is the nature of the voluntary forgetting processes? Ss described this phenomenon subjectively as if they were "looking through things." They saw the sỳ.s, but forgot them immediately afterward.

From the experimental results reported here, it is unlikely that the "blocking" hypothesis can be 
interpreted as reflecting non-rehearsal. Although several theorists, who offer duplexity models of memory (Waugh \& Norman, 1965; Atkinson \& Shiffrin, 1968, 1971), have suggested that rehearsal has a dual function of maintaining items in the STS and of transferring them to the LTS, other experiments did not show that this is necessarily so (Tulving, 1966; Glanzer \& Meinzer, 1967). The transmission of information from the STS to the LTS could be controlled by other processes, e.g., organization, imagery, or even by a simple blocking mechanism between these two stores. For example, Eagle and Ortof (1967) suggested that reduction in attention paid to the perceived stimuli blocks its further processing (also see Craik \& Lockhart, 1972).

\section{REFERENCES}

Atkinson, R. C., \& Shiffrin, R. M. Human mem ory: a proposed system and its control processes. In K. W. Spence and J. T. Spence (Eds.) The psychology of learning and motivation: Advances in Research and Theory, Vol. II, New York: Acad emic Press, 1968.

Atkinson, R. C., \& Shiffrin, R. M. The control of short-term memory. Scientific American, 1971, 224, 2, 82-89.

Bjork, R. A. Theoretical implications of directed forgetting. In A. W. Melton \& E. Martin (Eds.) Coding processes in human memory. Washington, D.C.: Winston \& Sons, 1972.

Bjork, R. A., LaBerge, D., \& LeGrande, R. The modification of short term memory through instructions to forget. Psychonomic science, 1968, 10, 55-56.
Block, R. A. Effects of instructions to forget in short-term memory. Journal of Experimental Psychology, 1971, 89, 1-9.

Craik, F.I.M., \& Lockhart, R. S. Levels of processing: a framework for memory research. Journal of Verbal Learning \& Verbal Behavior, 1972, 11,671-684

Eagle, M., \& Ortoff, E. The effect of level attention upon "phonetic" recognition errors. Journal of Verbal Learning \& Verbal Behavior, 1967, 6, 226-231.

Epstein. W. Mechanisms of directed forgetting. In G. H. Bower (Ed.), The psychology of learning and motivation, Vol, 6 . New York: Academic Press, 1972

Epstein, W., Massaro, D. W., \& Wilder, L. Selective search in directed forgetting. Journal of Experimental Psychology, $1972,94,18-24$

Epstein, W., \& Wilder, L. Searching for to-be-forgotten material in a directed forgetting task. Journal of Experimental Psychology, 1972, 95, 349-357

Glanzer, M. \& Meinzer, A. The effects of intralist activity on free recall. Journal of Verbal Learning and Verbal Behavior, 1967. $6,928-935$.

Noble. C. E., Stockwell, F. E., \& Pryer, M. W. Meaningfulness $\left(\mathrm{m}^{\prime}\right)$ and association value in paired-associate syllable learning. Psychological Reports, 1957, 3, 441-452.

Shebilske, W., Wilder, L., \& Epstein, W., Forget instructions: the effect of selective rehearsal and categorical distinctiveness. Journal of Experimental Psychology, 1971, 89, 372-378.

Tulving, E. Subjective organization and effects of repetition in multi-trial free-recall learning. Journal of Verbal Learning \& Verbal Behavior, 1966, 5, 193-197.

Underwood, B. J. \& Schulz, R. W. Meaning fulness and verbal learning. New York: Lippincott, 1960.

Waugh, N. C. \& Norman, D. A. Primary memory. Psy chological Review, 1965, 72, 89-104.

Winer, B. J. Statistical principles in experimental design. 2nd ed. New York: McGraw-Hill, 1971.

(Received for publication September 28, 1973; revision accepted May 26,1974 .) 